Polymers of Ministry of Education, Department of Chemistry, Wuhan University; ${ }^{3} \mathrm{Key}$ Laboratory of Biomedical Polymers of Ministry of Education, Department of Chemistry, Wuhan University, Wuhan 430072, P. R. China; ${ }^{4}$ Department of Ultrasonography, Renmin Hospital of Wuhan University

Objectives Previous studies have exhibited the protective effects of synthesised polymers alone oras scaffold for myocardial infractions (MI) treatment. However, the exact roll of synthesised polymers when used for MI treatment is still unknown. Besides that, the suitable degradation time of synthesised biomaterilas is still undercontroversy. In our present study, we synthetise two kinds of poly $\mathrm{N}$-isopropylacrylamide thermoresponsive hydrogel with theoretic different degradation time, and aim to investigate the interaction of physical characteristics and function of the synthesised polymerin MIinduced rat model.

Methods Twotypes of poly $\mathrm{N}$-isopropylacrylamide thermoresponsive hydrogel (defined as Gel A and GelB) were synthesised by our previous report. In vivo hydrogel formation and maintenance were observed and confirmed in male KM mice $(20 \pm 5 \mathrm{~g})$ to make sure the degredation days of hydrogel in vivo. MI was induced in male Wistar rats (200 $\pm 20 \mathrm{~g}$ ) by the ligation of left anterior descending conronary artery. The MI rats were grouped to receive intra-myocardialinjection of $100 \mu \mathrm{l}$ phosphate buffered saline, $3 \%$ (w/v) GelA or 3\% (w/v) Gel B solution randomly by intra-myocardium injection. The geometric structure of the hydrogel was also test by electron scanning microscopy. Echocardiographyand hemodynamic analysis were used to evaluate left ventricular systolic and diastolic function. Isolated infarcted or sham myocardium was tailored for contractility measurement in vitro. Western blotting and immunohistochemistry were used for detecting collagen metabolism.

Results In vivo degradation investigation showed the biodegradation time of Gel $\mathrm{A}$ is approximate 85 days and the days of Gel B is nearly 28 days. Electron scanning microscopy images exhibited the threedimensional structure of cross-linked and skeleton-liked networks contributing to form holes which could adhere to red blood cells. At the aspect of inhibiting ventricular infarction enlargement, Gel A or Gel B showed no significant difference, while, Gel B performed better than Gel A on improving left ventricular systolic and diastolic function $(p<0.05)$. Collagen hyper-deposition around the infarcted and noninfarcted myocardium is a common phenomenon after infarction. However, the injection of thermoresponsive hydrogel could significantly down-regulated collagen deposition. Notably, the effectiveness of hydrogel on regulate collagen metabolism is not through TFG- $\beta$ signal pathway. Additionally, contractitility of isolated infarcted myocardium was significantly deteriorated, however, contractionforce significant increased due to the improvement of contractive ordination after hydrogel used.

Conclusions Synthesised poly $\mathrm{N}$-isopropylacrymide hydrogel is an excellent option for MI treatment. Properphysical featrues may be the potential reason for the effective cardiacprotection of the thermoresponsive hydrogel post-MI. Additionally, suitabledegradation time of synthesised poly $\mathrm{N}$-isopropylacrymide hydrogel is a anotherconsideration for biomaterials designing. We deem the physical properties of polyN-isopropylacrymide hydrogel promote its effects on structural and functional replacement of damaged myocardium tissue.

\section{GW23-e0905 PHYSICAL FEATURES OF THERMORESPONSIVE HYDROGELS PROMOTE ITS EFFECTS ON CARDIAC PROTECTION POST MYOCARDIAL INFARCTION}

doi:10.1136/heartjnl-2012-302920b.3

\author{
${ }^{1}$ Shan Ren, ${ }^{1}$ Xuejun Jiang, ${ }^{2}$ Zeyong Li, ${ }^{1} Y$ Ying Wen, ${ }^{1}$ Dan Chen, ${ }^{1}$ Xiaoyan Li, \\ ${ }^{2}$ Xianzheng Zhang, ${ }^{3}$ Renxi Zhuo, ${ }^{4}$ Honggang Chu, ${ }^{1}$ Xuejun Jiang. ${ }^{1}$ Department of \\ Cardiology, Renmin Hospital of Wuhan University; ${ }^{2}$ Key Laboratory of Biomedical
}

